\title{
Sequence of the Variant Thyroxine-binding Globulin of Australian Aborigines Only One of Two Amino Acid Replacements is Responsible for Its Altered Properties
}

\author{
Kyoko Takeda," Yuichi Mori," Sylwester Sobieszczyk, " Hisao Seo," Matthew Dick," Frank Watson," Irwin L. Flink, ** \\ Susumu Seino," Graeme I. Bell, ${ }^{* 8}$ and Samuel Refetof ${ }^{* *}$ \\ Departments of ${ }^{*}$ Medicine, ${ }^{\ddagger}$ Pediatrics, ${ }^{\S}$ Biochemistry, and Molecular Biology and Howard Hughes Medical Institute, The University of \\ Chicago, Chicago, Illinois 60637; "Department of Endocrinology and Metabolism, The Research Institute of Environmental Medicine, \\ Nagoya, Japan; "Clinical Biochemistry Service, The Queen Elizabeth II Medical Center, Perth, Australia; \\ and ${ }^{* *}$ Departments of Medicine and Pharmacology, University of Arizona, Tuscon, Arizona 85719
}

\begin{abstract}
A form of thyroxine-binding globulin (TBG) with reduced affinity for hormone and increased susceptibility to heat and acid denaturation has been identified in Australian Aborigines (TBG-A). Results of heat denaturation of TBG established that the $\mathrm{TBG}_{\mathrm{A}}$ allele is $\mathrm{X}$ linked and has a frequency of $\mathbf{5 0 . 9 \%}$ in Western Australian Aborigines.

The sequence of an isolated $\mathbf{T B G}_{\mathbf{A}}$ allele differed at two positions from that of the normal TBG allele $\left(\mathrm{TBG}_{\mathrm{C}}\right)$. One substitution was in codon 191, ACA (threonine) rather than GCA (alanine), and the other was in codon 283, TTT (phenylalanine) instead of TTG (leucine). These nucleotide substitutions resulted in the loss of sites for the enzymes Bgl 1 and Tth 111 II, respectively. The nucleotide substitutions in the TBG-A allele were confirmed by digestion of genomic DNA segments amplified using the polymerase chain reaction. The Bgl 1 and Tth 111 II sites were absent in the genes of two Aboriginal men expressing TBG-A and were present in those of three Aboriginal and six Caucasian males expressing TBG-C. The TBG gene of a seventh Caucasian male possessed the Bgl 1 site but had lost the Tth 111 II site; sequencing of this allele revealed only the substitution in codon 283 identical to that in the TBG $_{A}$ allele. As the biochemical properties of $\mathrm{TBG}^{\mathrm{Phe}-283}$ expressed by this individual were indistin-

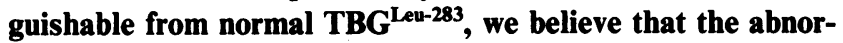
mal properties of TBG-A are due to substitution of alanine for threonine at residue 191.
\end{abstract}

\section{Introduction}

Thyroxine-binding globulin (TBG) ${ }^{1}$ is the major thyroid hormone carrier protein in human serum $(1,2)$. It is synthesized

This work was presented in part at the Annual Meeting of The Endocrine Society, New Orleans, LA, 8-11 June, 1988.

Address reprint requests to Dr. Samuel Refetoff, Thyroid Study Unit, Box 138, The University of Chicago, 5841 South Maryland Avenue, Chicago, IL 60637.

Received for publication 7 September 1988 and in revised form 23 November 1988.

1. Abbreviations used in this paper: IEF, isoelectric focusing; PCR, polymerase chain reaction; TBG, thyroxine-binding globulin; TBG-A, Aboriginal TBG; TBG-C, common type TBG; $T_{3}$, triiodothyronine; $\mathrm{T}_{4}$, thyroxine.

J. Clin. Invest.

(c) The American Society for Clinical Investigation, Inc. $0021-9738 / 89 / 04 / 1344 / 05 \quad \$ 2.00$

Volume 83, April 1989, 1344-1348 by liver parenchymal cells and secreted as a 54-kD glycoprotein $(3,4)$. Although TBG is not necessary for thyroid hormone action, alterations in its concentration produce proportional changes in the level of circulating thyroxine $\left(\mathrm{T}_{4}\right)$ and triiodothyronine $\left(\mathrm{T}_{3}\right)(1,2)$.

In 1980 , two groups of investigators $(5,6)$ noted low total $\mathrm{T}_{4}$ concentration in $\sim 40 \%$ of healthy Australian Aborigines. Dick and Watson attributed this finding to the presence of a variant TBG (7). Subsequently, we confirmed their hypothesis by showing that, compared with the common type TBG (TBG-C), this variant TBG (TBG-A) has decreased affinity for $T_{4}$ and $T_{3}$ and increased susceptibility to denaturation by heat and acid but normal immunoreactivity and microheterogeneity on isoelectric focusing (IEF) (8). The inheritance of TBG-A was X-linked (9) as expected from the recent demonstration that a single TBG gene is located at Xq 21-22 (10).

We now present the amino acid sequence of TBG-A deduced by sequencing of this variant TBG gene. Nucleotide substitutions were found in the codons for amino acids 191 and 283 resulting in replacement of alanine for threonine and leucine for phenylalanine, respectively. Both substitutions were present in the TBG genes of two unrelated Aboriginal men expressing TBG-A but were absent in the TBG genes of three Aboriginal men expressing TBG-C. Interestingly, an identical nucleotide substitution was found in the codon for amino acid 283 in the TBG gene of a Caucasian man, expressing a protein with properties indistinguishable from TBG-C. It thus is likely that the threonine substitution at amino acid 191 is responsible for the altered properties of TBG-A.

\section{Methods}

Subjects. Serum samples were obtained from 68 unrelated euthyroid Aborigines, 34 men and 34 women, from Western Australia. Their mean ages \pm SD were $34.9 \pm 13$ yr (range: $16-60$ ) and $35.5 \pm 17$ yr (range: 13-78), respectively. These samples were used to determine the prevalence of TBG-A in this population. In addition, serum samples and whole blood collected in EDTA, were obtained from five Aboriginal men, two known to have TBG-A (subjects 1 and 2) and three expressing the common protein, $\mathrm{TBG}_{\mathrm{C}}$ (subjects 3,4 , and 5). Sera and whole blood were also obtained from seven Caucasian men having TBG-C. Sera were kept frozen at $-20^{\circ} \mathrm{C}$ and whole blood was kept frozen at $-80^{\circ} \mathrm{C}$ until analysis of TBG and DNA extraction, respectively, except during the 48-h period of shipment in dry ice from Western Australia to Chicago.

Tests of thyroid function. The serum concentrations of total $\mathrm{T}_{4}$, total $\mathrm{T}_{3}$, and thyrotropin (TSH) were measured in serum by standard RIAs. TBG concentration in serum was determined by a specific RIA measuring native TBG as previously described (11).

Typing of TBG. The presence of TBG-A, TBG-C, or both TBG-A and TBG-C in heterozygous subjects, was determined by their rate of inactivation at $56^{\circ} \mathrm{C}$ as previously described (12). Briefly, residual na- 


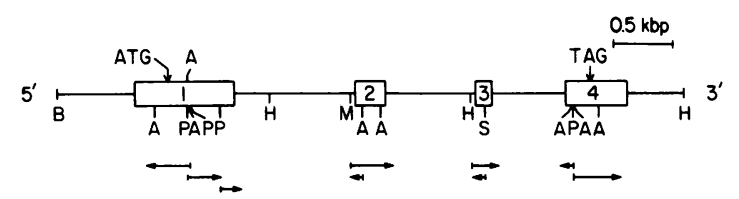

Figure 1. Schematic representation of the TBG gene and strategy of sequencing. Exons 1-4 are denoted by open boxes. Translational initiation (ATG) and termination (TAG) codons are indicated. Only those restriction endonuclease sites relevant to subcloning into $M 13$ phage for sequencing are shown (A, Alu I; B, Bam HI; H, Hind III; M, Msp I; P, Pst I; S, Sph I). Arrows indicate the regions sequenced.

tive (nondenatured) TBG was measured by RIA in diluted serum samples before and 20,40 , and $60 \mathrm{~min}$ after exposure to $56^{\circ} \mathrm{C}(11,12)$. The $t_{1 / 2}$ of TBG denaturation was determined by the method of leastsquares applied to the best fitting straight line of the logarithm of residual native TBG concentrations and the corresponding times of incubation analyzed. Data were expressed as percent of control to correct for intraassay variation and small $\left(0.2^{\circ} \mathrm{C}\right)$ but significant temperature variations during batch analysis. Sera from five Caucasians containing TBG-C were included in each analysis. All samples were submitted to IEF and the pattern of TBG microheterogeneity was determined as previously described (12).

Isolation and sequencing of TBG genes. Unless otherwise specified, recombinant manipulations were carried out using standard protocols (13). Restriction enzymes were purchased from either Bethesda Research Laboratories, Inc. (Gaithersburg, MD) or New England Nuclear (Boston, MA) and used as suggested by the suppliers.

DNA was isolated from the nuclei of peripheral blood lymphocytes as previously described (14) and was digested with Eco RI. Resultant fragments of 10-20 kb, which included the 14-kb fragment containing the TBG gene, were isolated by electrophoresis in a $0.8 \%$ low meltingtemperature agarose gel and then ligated into the Eco RI arms of $\lambda$ EMBL4. After packaging and infection of Escherichia coli strain LE 392 (Stratagene Corp., San Diego, CA), recombinant phages containing the TBG gene were identified by hybridization with a human liver TBG cDNA probe ( $\lambda$ cTBG-8, reference 15 ) labeled with ${ }^{32} \mathrm{P}$ by nick translation (kit; Amersham Corp., Arlington Heights, IL). Appropriate restriction fragments of the TBG gene (Fig. 1), were subcloned into M13mp18 or M13mp19 and sequenced by the dideoxynucleotide chain termination method (16).

Amplification of specific gene segments and their analysis. Exons 2 and 3 of the TBG gene containing the mutation sites were amplified by the polymerase chain reaction (PCR) method. Oligonucleotides A, B, $C$, and D (Table I and Fig. 2), serving as primers, were synthesized on a DNA Synthesizer (model 308 B; Applied Biosystems, Foster City, CA) by the $\beta$-cyanoethyl chemistry.

PCR was carried out by a modification of the method described by Saiki et al. (17). $1.5 \mu \mathrm{g}$ of undigested genomic DNA served as the initial template for each pair of primers, 100 pmol of which were added

Table I. Sequences and Locations of Oligonucleotide Primers

\begin{tabular}{ccc}
\hline Primer & Sequence & Location \\
\hline A & $5^{\prime}$-CTTGGCATATTCTAGTGATC-3' & 136 nucleotides \\
& & $3^{\prime}$ from exon 2 \\
B & $5^{\prime}$-CCTTGATCCTCAGTCACTTG-3' & 151 nucleotides \\
& & $5^{\prime}$ from exon 2 \\
C & $5^{\prime}$-TCAGAACTGCATCTCACCA-3' & 268 nucleotides \\
& & $3^{\prime}$ from exon 3 \\
D & $5^{\prime}$-AAGCTTGATATGGTGATTGC-3' & 48 nucleotides \\
& & $5^{\prime}$ from exon 3
\end{tabular}

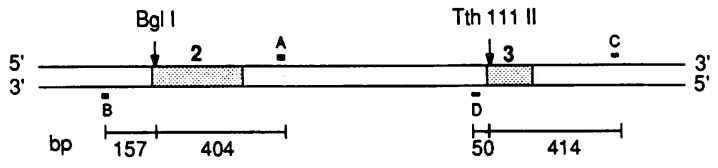

Figure 2. Strategy of amplication of exons 2 and 3 by PCR and analysis using restriction endonucleases. Exons 2 and 3 are indicated. A, B, C, and D are primers used for PCR (Table I) positioned at binding sites. Bars with numbers represent fragment sizes generated by digestion of the amplified TBG-C DNA segments with the indicated restriction endonucleases, whose sites of cleavage are shown by arrows.

together with 2.5 U Thermus aquaticus DNA polymerase (PerkinElmer Cetus Corp., Norwalk, CT). The $100-\mu l$ reaction mixture also contained $1.25 \mathrm{mM}$ of each deoxynucleotide triphosphate (dATP, dCTP, dGTP, and TTP), and $10 \mathrm{mM}$ Tris- $\mathrm{HCl}, 50 \mathrm{mM} \mathrm{KCl}, 1.5 \mathrm{mM}$ $\mathrm{MgCl}-2$, and $0.01 \%$ gelatin, $\mathrm{pH}$ 8.3. The reaction mixture was covered with $100 \mu \mathrm{l}$ mineral oil (Sigma Chemical Co., St. Louis, MO) and submitted to amplification using a DNA thermalcycler (Perkin-Elmer Cetus Corp.). The initial template denaturation was at $94^{\circ} \mathrm{C}$ for 1.5 min. Afterwards, a typical cycle profile was $2.5 \mathrm{~min}$ at $55^{\circ} \mathrm{C}$ for annealing, $3.5 \mathrm{~min}$ at $65^{\circ} \mathrm{C}$ for extension, and $1 \mathrm{~min}$ at $94^{\circ} \mathrm{C}$ for denaturation. After 30 such cycles, the reaction mixtures containing the amplified DNA segments (Fig. 2) were extracted with chloroform to remove the mineral oil and $10 \mu \mathrm{l}$ of each was digested separately with either Bgl I and Tth $111 \mathrm{II}$. To remove DNA binding proteins present in the Tth $111 \mathrm{II}$ preparation and which interfere with the subsequent electrophoretic separation, it was necessary to extract these samples twice with an equal volume of buffer ( $50 \mathrm{mM}$ Tris- $\mathrm{HCl}, 1 \mathrm{mM}$ EDTA and $0.1 \% \beta$ mercaptoethanol, $\mathrm{pH} 8.0$ ) saturated phenol and once with an equal volume of chloroform/isoamyl alcohol (24:1) and then precipitate with $100 \%$ ethanol. DNA fragments were resolved by electrophoresis in a $2 \%$ GTG agarose gel (NuSieve; FMC BioProducts, Rockland, ME). The separated DNA fragments were visualized with ultraviolet light after staining with ethidium bromide.

\section{Results}

The prevalence of $T B G-A$. The rates of TBG denaturation in serum samples from the 68 unrelated Australian Aborigines are shown in Fig. 3. Those of the 34 Aboriginal men segregated into either of two distinct groups, one ranging from 94 to $125 \%$

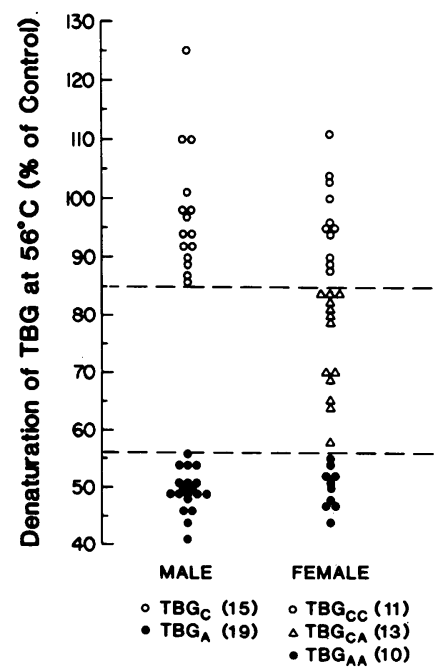

Figure 3. Typing of TBG in serum by the rate of its denaturation at $56^{\circ} \mathrm{C}$. Results are expressed as percent of the mean $t_{1 / 2}$ of denaturation of TBG-C from Caucasians analyzed in the same assay. In parentheses are the number of individuals of the indicated phenotype according to sex. 
Table II. Prevalence of TBG-A and TBG-C and Allele Frequency in Aborigines

\begin{tabular}{llcclll}
\hline & \multicolumn{3}{c}{ Phenotype (No.) } & & \multicolumn{2}{c}{ Allele (No.) } \\
\cline { 2 - 3 } \cline { 7 - 8 } & A & AC & C & & A & C \\
\hline Men & 19 & 0 & 15 & & 19 & 15 \\
Women & 10 & 13 & 11 & & 33 & 35 \\
Total & 29 & 13 & 26 & & 52 & 50 \\
$\quad(\%)$ & 42.6 & 19.1 & 38.3 & & 50.9 & 49.1 \\
Expected (\%) & 38.4 & 25.0 & 36.6 & &
\end{tabular}

* Calculated by the formula of Hardy-Weinberg, assuming a TBG $_{A}$ allele frequency of $50.9 \%$ and $\mathrm{X}$-linked inheritance.

of mean Caucasian controls and the other from 56 to $41 \%$ of control values. By contrast, the rates of heat denaturation of TBG in samples from Aboriginal women distributed as a continuum spanning the extreme values observed in men (43 to $111 \%$ of control). The lowest value in males with normal rates of TBG denaturation ( $56 \%$ of control) and the highest value in males with rapid rates of TBG denaturation ( $85 \%$ of control) served as cut-off points to identify heterozygous women expressing both TBG-A and TBG-C.

Based on these results, the prevalence of TBG-A was determined (Table II). The prevalence of hemizygous men with $\mathrm{TBG}_{\mathrm{A}}$ was $55.9 \%$ and that for men with TBG-C was $44.1 \%$. $29.4 \%$ of women were homozygous for TBG-A, $32.4 \%$ were homozygous for TBG-C, and the remaining $38.2 \%$ were heterozygotes. This distribution is in good agreement with that expected based on Hardy-Weinberg equilibrium, the $\mathrm{TBG}_{\mathrm{A}}$ allele frequency being $50.9 \%$.

The IEF pattern (not shown) of TBG was determined in all samples, and as previously shown (8), no differences were observed between TBG-A and TBG-C.

Sequence of $T B G-A$. The sequences of the coding regions and adjacent introns of a TBG-A allele from subject 1 (Table III) were compared with those of an allele isolated from an Aboriginal man expressing a TBG with the properties of TBG-C (subject 3 in Table III). The nucleotide sequence of this latter allele was identical to that of three alleles isolated from Caucasian men expressing TBG-C. The sequence of the
$\mathrm{TBG}_{\mathrm{A}}$ allele differed at sites in exons 2 and 3 and were verified by sequencing both strands: a G-to-A transition in the codon for amino acid 191 of the mature protein in exon 2 resulted in the substitution of alanine (GCA) with threonine (ACA), and a G-to-T transversion in the codon for amino acid 283 in exon 3 replaced leucine (TTG) with phenylalanine (TTT). These nucleotide substitutions result in the loss of the recognition sites for $\mathrm{Bgl} I\left[\mathrm{GCC}(\mathrm{N})_{5} \mathrm{GGC} \rightarrow \mathrm{GCC}(\mathrm{N})_{5} \mathrm{GAC}\right]$ and Tth 111 II (TGPyTTG $\rightarrow$ TTPyTTG) in exons 2 and 3, respectively (Fig. 4). The mutation in exon 2 was 9 nucleotides downstream from the splice junction with exon 1 (Ala-188, G|CC) and that in exon 3, 13 nucleotides downstream from the splice junction with exon 2 (Gly-279, GG|A).

Identification of TBG-A type substitutions by exon amplification and digestion with restriction endonucleases. To determine whether one or both substitutions are responsible for the observed changes in the properties of TBG-A, we exploited the loss of the recognition sites for Bgl I in exon 2 and Tth 111 II in exon 3 to screen available DNA samples. Exons 2 and 3 from five Aboriginal men (two with TBG-A and three with TBG-C, Table III) and seven Caucasian men with TBG-C were amplified by the PCR (Fig. 2) and digested with Bgl I and Tth 111 II, respectively. As shown in Fig. 5, the Bgl I and Tth 111 II sites were absent in the amplified DNA samples containing exon 2 (561 bp) and exon 3 (464 bp), respectively, generated from two Aboriginal men expressing TBG-A. In contrast, amplified segments of DNA from the three Aboriginal men expressing TBG-C were digested as predicted yielding DNA segments of 404 and 157 bp with $\mathrm{Bgl} I$ and a 414-bp segment with Tth $111 \mathrm{II}$. The 50-bp segment resulting from the latter digestion was not visualized on this agarose gel; furthermore, because digestion with this endonuclease was incomplete, both the original 464-bp segment and the 414-bp fragment of the Tth 111 II digested samples are evident; both fragments hybridized with a TBG-C DNA probe (not shown). The TBG typing of the five Aboriginal men using this procedure was in complete agreement with results of typing based on both heat inactivation and serum $\mathrm{T}_{4}$ and $\mathrm{T}_{3}$ concentrations (Table III). The amplified DNA segments of the Aboriginal men expressing TBG-A (subject 2 in Table III) and that of another Aboriginal man expressing $\mathrm{TBG}_{\mathrm{C}}$ (subject 4 in Table III) whose TBG genes were not cloned, were sequenced, confirming the results from specific endonucleases digestion.

Table III. Typing of Serum TBG from Men Whose DNA Was Analyzed

\begin{tabular}{|c|c|c|c|c|c|c|c|c|}
\hline \multirow[b]{2}{*}{ Origin } & \multirow[b]{2}{*}{ TBG Type } & \multirow[b]{2}{*}{ Subject No. } & \multirow[b]{2}{*}{$\mathrm{T}_{4}$} & \multirow[b]{2}{*}{$\mathrm{T}_{3}$} & \multirow[b]{2}{*}{ TSH } & \multicolumn{3}{|c|}{ TBG } \\
\hline & & & & & & & & Concentration \\
\hline & & & $\mu g / d l$ & $n g / d l$ & $\mu U / m l$ & $\min$ & $\%$ of control & $m g / d l$ \\
\hline Aborigine & TBG-A & 1 & 2.2 & 31 & 0.7 & 23 & 51 & 0.76 \\
\hline Aborigine & TBG-A & 2 & 2.0 & 34 & 4.0 & 22 & 49 & 0.73 \\
\hline Aborigine & TBG-C & 3 & 10.3 & 145 & 4.4 & 41 & 90 & 1.72 \\
\hline Aborigine & TBG-C & 4 & 6.5 & 91 & 3.7 & 51 & 113 & 1.10 \\
\hline Aborigine & TBG-C & 5 & 7.2 & 77 & 3.1 & 46 & 102 & 1.16 \\
\hline Caucasian $^{\ddagger}$ & TBG-C & 6 & 7.8 & 118 & 2.4 & 54 & 120 & 1.60 \\
\hline Caucasian & TBG-C & 7 & 6.2 & 103 & 1.8 & 40 & 89 & 1.10 \\
\hline
\end{tabular}

* Heat denaturation at $56^{\circ} \mathrm{C} . \quad{ }^{\ddagger}$ Data from five additional Caucasian men with TBG-C are not shown. 


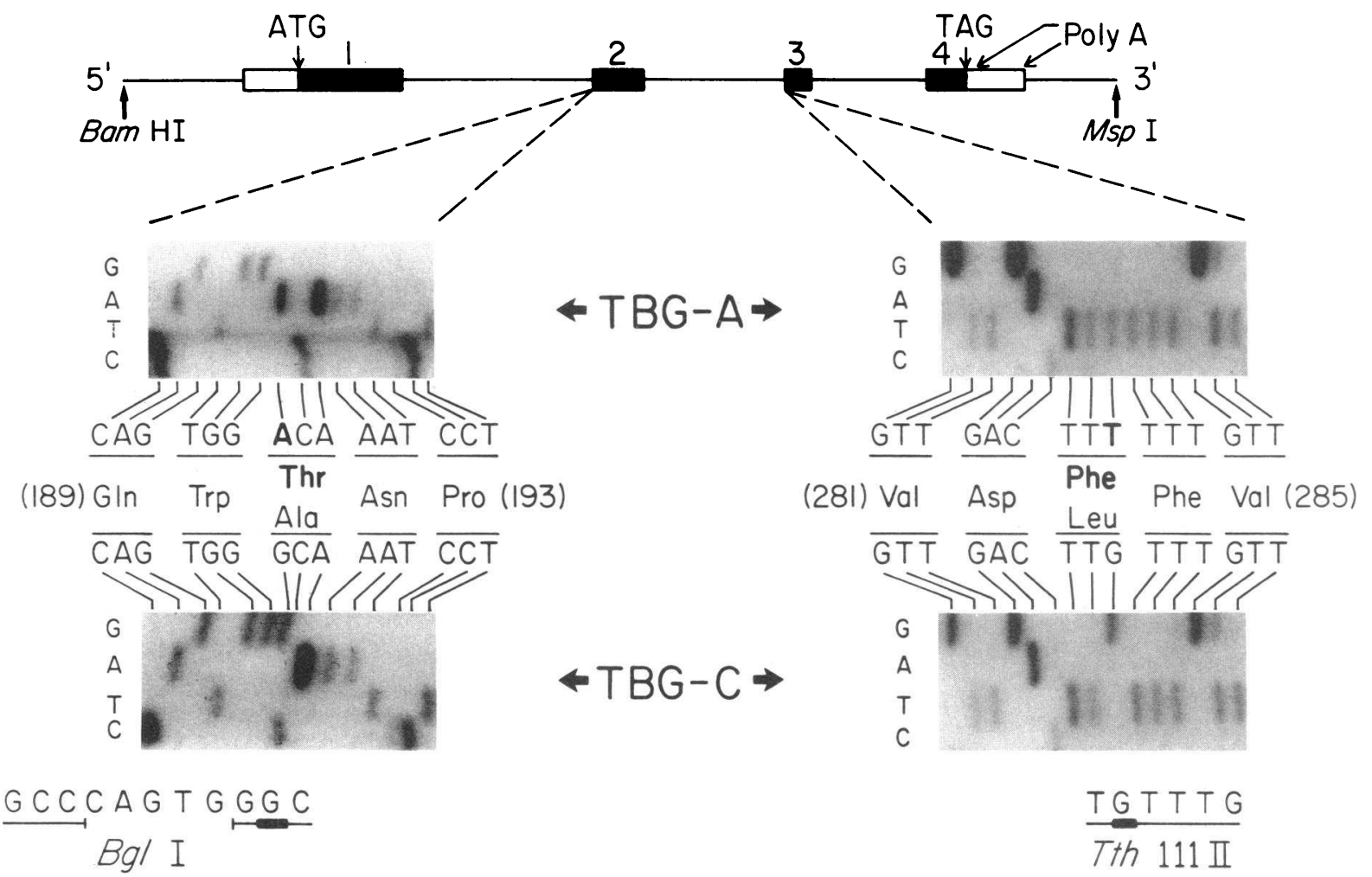

Figure 4. Structure of the TBG gene and sections of sequencing gels showing the substitutions in TBG-A. On top of the figure is the schematic representation of the gene with translated sequences $(\square)$, untranslated exon sequences $(\square)$, intervening and flanking sequences $(\longrightarrow)$. Initiation (ATG) and termination (TAG) codons and the alternative polyadenylation sites (Poly A) are indicated. Amino acid residues numbered consecutively from the $\mathrm{NH}_{2}$-terminus of the mature protein are shown in brackets. $\mathrm{G}$ in codon 191 of TBG-A is replaced by $A$ in TBG-C and G in codon 283 by $\mathrm{T}$. The resulting amino acid substitutions are indicated. The losses of restriction endonucleases recognition sites resulting from the two point mutations are also shown.

More revealing were the results obtained on the analysis of seven Caucasian men expressing TBG-C. Whereas exon 2 of each could be digested with Bgl I, one of the seven samples resisted digestion with Tth 111 II (Fig. 5, subject 7). The sequence of exon 3 of this subject revealed the same nucleotide substitution in the codon for residue 283 as that found in the Aboriginal men with TBG-A. However, the TBG synthesized by this individual had properties indistinguishable from those of TBG-C, including rate of heat denaturation and IEF pattern. Moreover, his serum $T_{4}$ and $T_{3}$ concentrations were within the normal range (Table III, subject 7).

\section{Discussion}

Measurement of the rate of TBG denaturation at $56^{\circ} \mathrm{C}$ provides a means of distinguishing not only TBG-A from TBG-C but also of identifying heterozygotes having both TBG-A and TBG-C (9). The demonstration of the $\mathrm{TBG}_{\mathrm{AC}}$ phenotype in 13 of 34 Aboriginal women and its absence in 34 Aboriginal men, is consistent with X-linked mode of inheritance of this gene. An earlier report suggesting an autosomal inheritance (18) was due to the inability to identify heterozygotes combined with the high frequency of the $\mathrm{TBG}_{\mathrm{A}}$ gene in Australian Aborigines. In the larger sample presented here, the frequencies of the $\mathrm{TBG}_{\mathrm{A}}$ and $\mathrm{TBG}_{\mathrm{C}}$ alleles are almost equal, 50.9 and $49.1 \%$, respectively. Because men have a single TBG gene and women have two alleles, in the mixed population $29(42.7 \%)$ had in serum only TBG-A, 26 (38.2\%) had only TBG-C, and 13 (19.1\%) had both TBG-A and TBG-C.

Of the two amino acid replacements in TBG-A, the substitution of phenylalanine for leucine-283 represents a relatively conservative replacement and thus is unlikely to be responsible for the abnormal properties of TBG-A. Moreover, we also observed the same substitution in one of seven Caucasian men examined, in whom it was not associated with detectable changes in the properties of the TBG molecule. Thus, it seems likely that the Leu/Phe-283 represents a common polymorphism that antedates the mutation at amino acid 191. The latter, present in both Aboriginal men with TBG-A examined, results in the substitution of a polar threonine for the nonpolar alanine-191.

The data strongly suggest that the threonine-191 substitution is responsible for both abnormal binding affinity and stability of TBG-A. What remains unclear is whether it is solely responsible for the observed alterations or whether the phenylalanine-283 is also required. As the threonine-191 mutation likely occurred on a chromosome having phenylalanine at residue 283, it is unlikely that the analysis of a larger group of Aboriginal men with TBG-A will resolve this quandary. A more direct approach would require the in vitro expression in cultured cells of variants having one and both substitutions.

Finally, we would propose changing the eponym, TBG-A, for $\mathrm{TBG}^{\mathrm{Thr}-191, \mathrm{Phe}-283}$ and to designate the TBG with properties indistinguishable from TBG-C, TBG ${ }^{\text {Phe-283 }}$. 


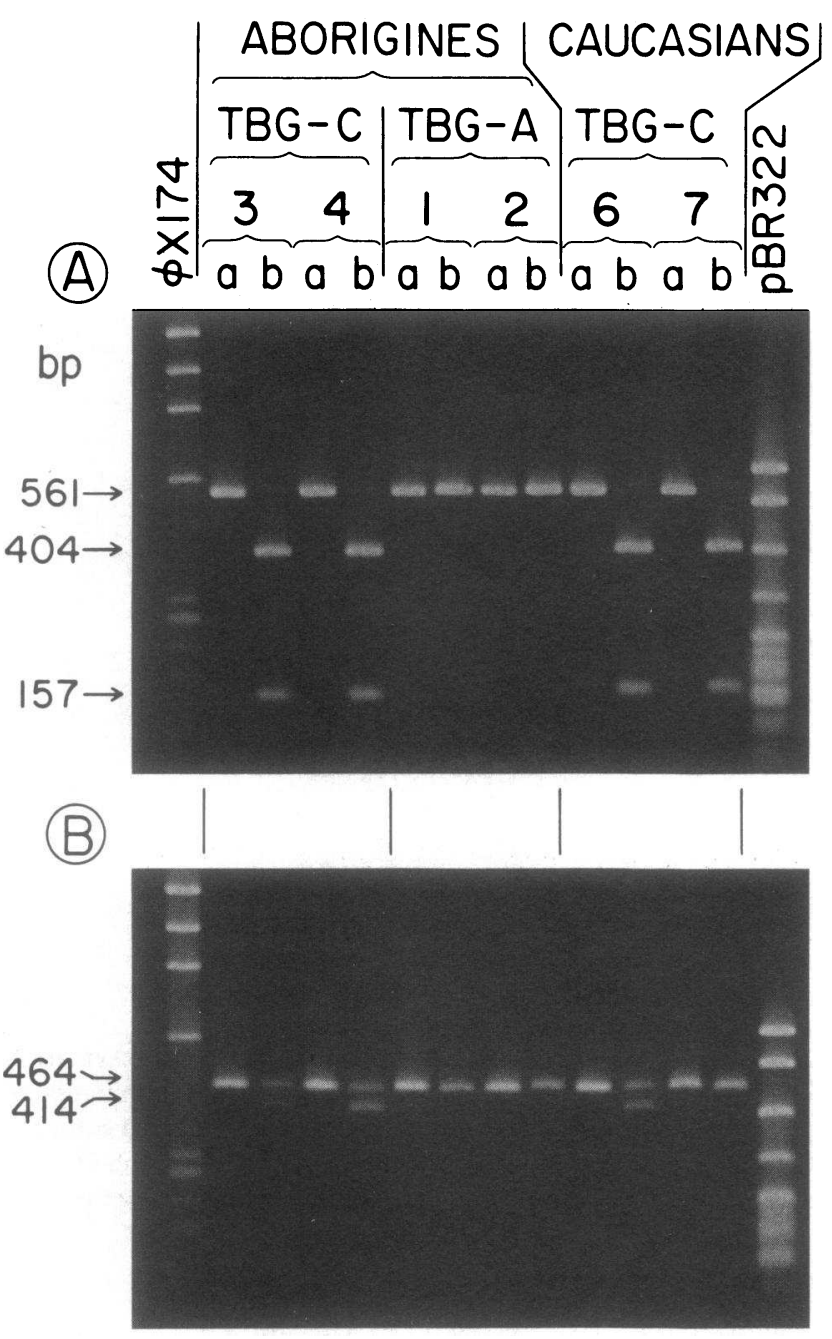

Figure 5. Detection of nucleotide substitutions by restriction endonuclease digestion of amplified DNA sequences. Numbers identifying lanes correspond to the subjects described in Table III. $a$ and $b$ denote undigested and digested samples, respectively. $(A)$ Digestion of the amplified DNA segments containing exon 2 (561 bp) with $\mathrm{Bgl}$ I generated two fragments of 404 and 157 bp in all DNA samples, except those from Aboriginal men expressing TBG-A. (B) Digestion of the amplified DNA segments containing exon 3 (464 bp) with Tth 111 II should generate two fragments of 414 and $50 \mathrm{bp}$ in TBG-C. The 50-bp fragment was not visualized and incomplete digestion (see Methods) resulted in two fragments, the undigested fragment of 464 bp and a 414-bp fragment. The three DNA samples from Aboriginal men with TBG-C (only two shown) and seven of eight samples from Caucasian men with TBG-C (only one is shown, subject 6) were digested by the enzyme. DNA samples from the Aboriginal men with TBG-A and from a Caucasian man with TBG-C (subject 7) were not digested.

\section{Acknowledgments}

We wish to thank Dr. Neal H. Scherberg for the measurement of $T_{4}$ and $\mathrm{T}_{3}$ in serum, Mr. Paul Gardner for the synthesis of oligonucleotide primers, Mr. Richard Grandoni and Miss Ann Flynn from New England Nuclear for the generous gift of Tth 111 II, Dr. Roy Weiss, Dr. E. Timothy Shapiro, and Dr. Theodore Pullman for their helpful comments on this manuscript, and Mrs. Yolanda W. Richmond for its preparation.
This work was supported in part by U. S. Public Health Service grant DK-15070.

\section{References}

1. Robbins, J., and L. Bartalena. 1986. Plasma transport of thyroid hormone. In Thyroid Hormone Metabolism. G. Henneman, editor. Marcel Dekker, Inc., New York. 3-38.

2. Refetoff, S. 1979. Thyroid function tests. In Endocrinology. Vol. 1. L. J. DeGroot, editor, Grune \& Stratton, Inc., New York. 387-428.

3. Bartalena, L., and J. Robbins. 1984. Effect of tunicamycin and monensin on secretion of thyroxine-binding globulin by cultured human hepatoma (Hep G2) cells. J. Biol. Chem. 259:13610-13614.

4. Murata, Y., D. H. Sarne, A. L. Horwitz, R. Lecocq, D. P. Aden, B. B. Knowles, and S. Refetoff. 1985. Characterization of thyroxinebinding globulin secreted by a human hepatoma cell line. J. Clin. Endocrinol. \& Metab. 60:472-478.

5. White, G. H., and R. Morice. 1980. Diagnostic biochemical tests in Aboriginals. Med. J. Aust. (Spec. Suppl.) 1:6-8.

6. Dick, M., and F. Watson. 1980. Prevalent low serum thyroxinebinding globulin level in Western Australian Aborigines. Med. J. Aust. 1:115-118.

7. Dick, M., and F. Watson. 1981. A possible variant of thyroxinebinding globulin in Australian Aborigines. Clin. Chim. Acta. 116:361-367.

8. Murata, Y., S. Refetoff, D. H. Sarne, M. Dick, and F. Watson. 1985. Variant thyroxine-binding globulin in serum of Australian Aborigines: its physical, chemical and biological properties. J. Endocrinol. Invest. 8:225-232.

9. Refetoff, S., and Y. Murata. 1985. X-chromosome-linked inheritance of the variant thyroxine-binding globulin in Australian Aborigines. J. Clin. Endocrinol. \& Metab. 60:356-360.

10. Trent, J. M., I. L. Flink, E. Morkin, P. Van Tuinen, and D. H. Ledbetter. 1987. Localization of the human thyroxine-binding globulin gene to the long arm of the X-chromosome (Xq21-22). Am. J. Hum. Genet. 41:428-435.

11. Refetoff, S., Y. Murata, G. Vassart, V. Chandramouli, and J. S. Marshall. 1984. Radioimmunoassays specific for the tertiary and primary structures of thyroxine-binding globulin (TBG): measurement of denatured TBG in serum. J. Clin. Endocrinol. \& Metab. 59:269-277.

12. Takamatsu, J., S. Refetoff, M. Charbonneau, and J. Dussault. 1987. Two new inherited defects of the thyroxine-binding globulin (TBG) molecule presenting as partial TBG deficiency. J. Clin. Invest. 79:833-840.

13. Maniatis, T., E. F. Fritsch, and J. Sambrook. 1982. Molecular Cloning: A Laboratory Manual. Cold Spring Harbor Laboratory, Cold Spring Harbor, NY.

14. Bell, G. I., J. H. Karam, and W. J. Rutter. 1981. Polymorphic DNA region adjacent to the $5^{\prime}$ end of the human insulin gene. Proc. Natl. Acad. Sci. USA. 78:5759-5763.

15. Flink, I. L., T. J. Bailey, T. A. Gustefson, B. E. Markham, and E. Morkin. 1986. Complete amino acid sequence of human thyroxine-binding globulin deduced from cloned DNA: close homology to the serine antiproteases. Proc. Natl. Acad. Sci. USA. 83:7708-7712.

16. Sanger, F., S. Nicklen, and A. R. Coulson. 1977. DNA sequencing with chain terminating inhibitors. Proc. Natl. Acad. Sci. USA. 74:5463-5467.

17. Saiki, R. K., D. H. Gelfand, S. Stoffel, S. J. Scharf, R. Higuchi, G. T. Horn, K. B. Mullis, and H. A. Erlich. 1988. Primer-directed enzymatic amplification of DNA with thermostable DNA polymerase. Science (Wash. DC). 239:481-491.

18. Watson, F., and M. Dick. 1980. Distribution and inheritance of low serum thyroxine-binding globulin levels in Australian Aborigines. Med. J. Aust. 2:385-387. 